EPJ Web of Conferences 107, 05009 (2016)

DOI: 10.1051/epjconf/201610705009

C) Owned by the authors, published by EDP Sciences, 2016

\title{
On the excitation energy of deep-hole states in medium-heavy-mass spherical nuclei
}

\author{
G. V. Kolomiytsev ${ }^{1, a}$, S. Yu. Igashov ${ }^{1, b}$, and M. H. Urin ${ }^{1, c}$ \\ ${ }^{1}$ National Research Nuclear University "MEPhl” (Moscow Engineering Physics Institute), Moscow, Russia
}

\begin{abstract}
Within the particle-hole dispersive optical model it is shown that the spreading effect determines a significant part of the anomalously large excitation energy of deep-hole states in the ${ }^{90} \mathrm{Zr}$ and ${ }^{208} \mathrm{~Pb}$ parent nuclei.
\end{abstract}

\section{Introduction}

An anomalously large excitation energy of deep hole states in the ${ }^{90} \mathrm{Zr}$ and ${ }^{208} \mathrm{~Pb}$ parent nuclei has been experimentally found by Vorobyov et al. [1]. In most Hartree-Fock calculations exploiting different versions of Skyrme-type

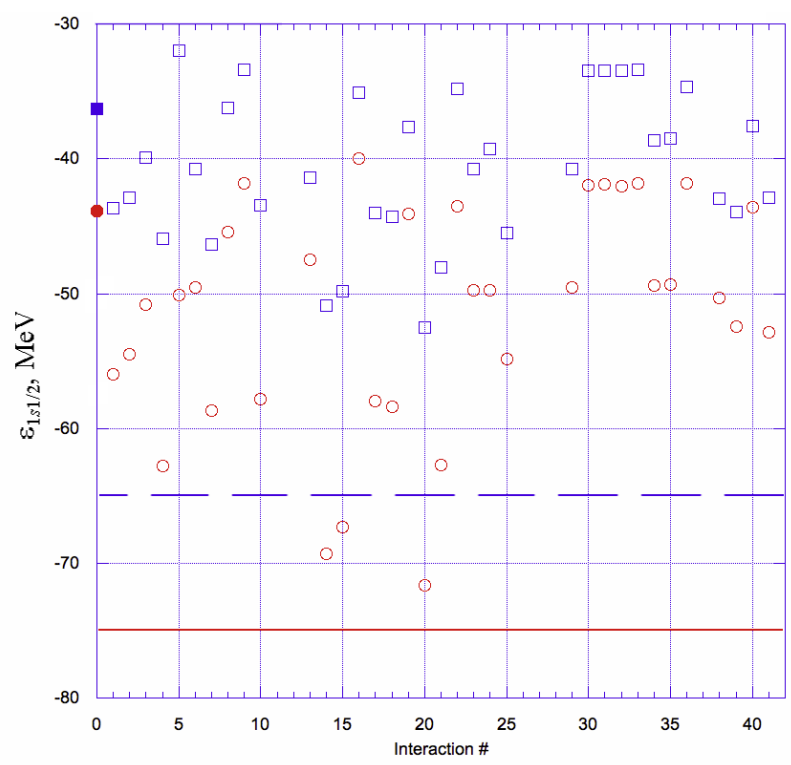

Figure 1. (Color online) The energies of $1 s_{1 / 2}$ hole states in the ${ }^{208} \mathrm{~Pb}$ parent nucleus obtained by Hartree-Fock calculations exploiting different versions of Skyrme-type forces (the circles and squares are corresponds to neutron and proton states, respectively) [2]; the filled symbols are related to the partially selfconsistent mean field (PSCMF) of Ref. [3]; the dashed and solid lines corresponds to the experimental data of Ref. [1] for proton and neutron states, respectively. The meaning of horizontal axis numbers is described in Table 1 .

\footnotetext{
ae-mail: flight160@gmail.com

be-mail: igashov@theor.mephi.com

$\mathrm{c}_{\mathrm{e}-\mathrm{mail}}$ : urin@theor.mephi.com
}

forces, the energy of deep-hole states in the ${ }^{208} \mathrm{~Pb}$ parent nucleus is markedly underestimated (Fig. 1). The same conclusion follows from calculations exploiting a realistic partially self-consistent phenomenological mean field provided that the parameters of this mean field adjusted to describe the observable single-quasiparticle spectra near the Fermi energy [3].

In the present work we attempt to show that the spreading effect due to coupling of deep-hole states to manyquasiparticle configurations contributes significantly to the deep-hole state excitation energy. To reach this aim, we use the single-quasiparticle dispersive optical model (SQDOM) formulated in a rather formal way long ago [4]. Microscopically-based transition to the SQDOM was performed recently [3].

Table 1. Variants of Skyrme forces provided by S. Shlomo [2] and used in calculations presented in Fig. 1.

\begin{tabular}{|c|c|l|c|l|c|}
\hline$\#$ & Interaction & $\#$ & Interaction & $\#$ & Interaction \\
\hline 0 & PSCMF & 14 & SkI3 & 28 & SkI6 \\
\hline 1 & KDE0 & 15 & SkI5 & 29 & SKM* \\
\hline 2 & KDE0v1 & 16 & SkP & 30 & SkT1 \\
\hline 3 & SGII & 17 & SLy5 & 31 & SkT2 \\
\hline 4 & SkI4 & 18 & SLy6 & 32 & SkT3 \\
\hline 5 & SK0 & 19 & SV-bas & 33 & SkT3* \\
\hline 6 & SKM $*$ & 20 & SV-m56-0 & 34 & SkT8 \\
\hline 7 & SkMP & 21 & SV-m64-0 & 35 & SkT9 \\
\hline 8 & SK0 & 22 & SV-min & 36 & Skxs20 \\
\hline 9 & SkT1 $*$ & 23 & LNS & 37 & SLy7 \\
\hline 10 & SLy4 & 24 & MSL0 & 38 & SQMC650 \\
\hline 11 & UNEDF0 & 25 & NRAPR & 39 & SQMC700 \\
\hline 12 & UNEDF1 & 26 & SAMi & 40 & SV-sym32 \\
\hline 13 & Sk255 & 27 & SIII & 41 & Z_sigma \\
\hline
\end{tabular}

This is an Open Access article distributed under the terms of the Creative Commons Attribution License 4.0, which permits unrestricted use, distribution, and reproduction in any medium, provided the original work is properly cited. 


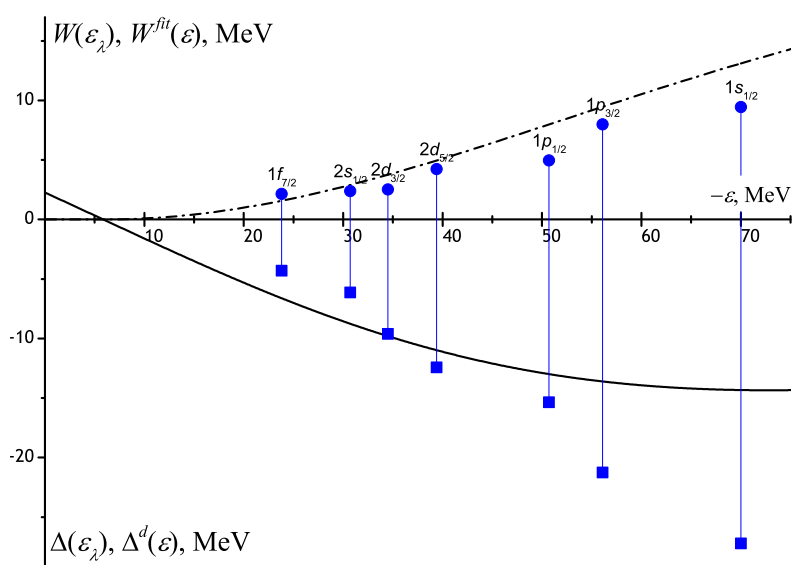

a)

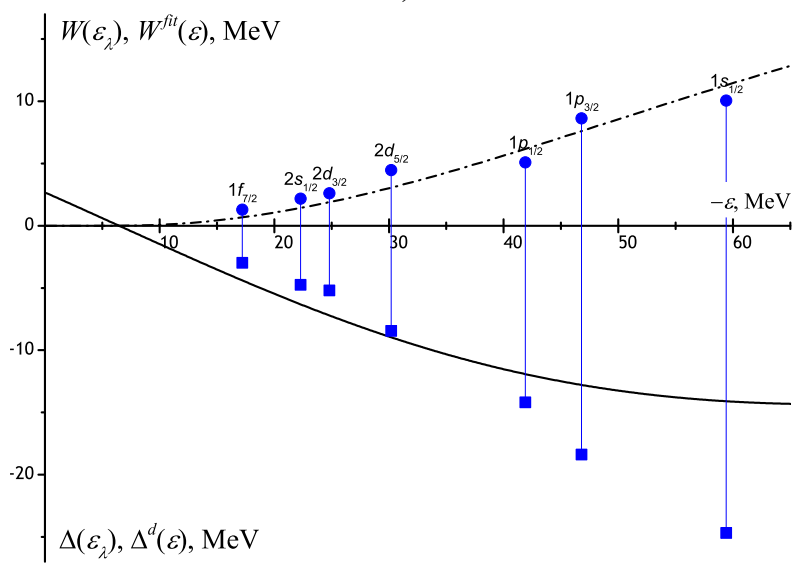

b)

Figure 2. (Color online) The quantities $\Delta\left(\varepsilon_{\lambda}\right)$ (squares) and $W\left(\varepsilon_{\lambda}\right)$ (circles) deduced from the experimental data of Ref. [1] with the use of Eqs. (1) and (2), respectively for the neutron (a) and proton (b) one-hole states in the ${ }^{90} \mathrm{Zr}$ parent nucleus. The adopted function $W^{\text {fit }}(\varepsilon)$ of Eq. (5) and the calculated function of Eq. (2) are shown by the dot-dashed and solid lines, respectively.

\section{Basic relations}

In the analysis we use, as the starting point, the phenomenological partially self-consistent mean field (described in details in Ref. [3]), which determines the singleparticle (s-p) Hamiltonian $H_{0}(x)$. Then the optical-model Hamiltonian is (the isobaric index, on which all considered quantities are diagonal, is omitted)

$$
H(x)=H_{0}(x)+[\mp i W(\varepsilon)+\Delta(\varepsilon)] f(x) .
$$

Here, the signs "_" and "+" are related to single-particle (s-p, $\varepsilon>\mu$ ) and single-hole (s-h, $\varepsilon<\mu$ ) excitations, respectively; $\mu$ is the mean (for particle and holes) chemical potential; $f(x)$ is the Woods-Saxon function used also in the definition of the mean field.

In the further use of Eq. (1) it is supposed the following: (i) the energy dependence of $W$ is similar for particles and holes, i.e. $W(\varepsilon)=W(|E|)$, where $E=\varepsilon-\mu$, and $|E|$ is the single-quasiparticle excitation energy; (ii) the real quantity $\Delta$ can be presented as the sum $\Delta^{d}+\Delta^{p}$, where the first ("dispersive") term is due to the spreading effect while

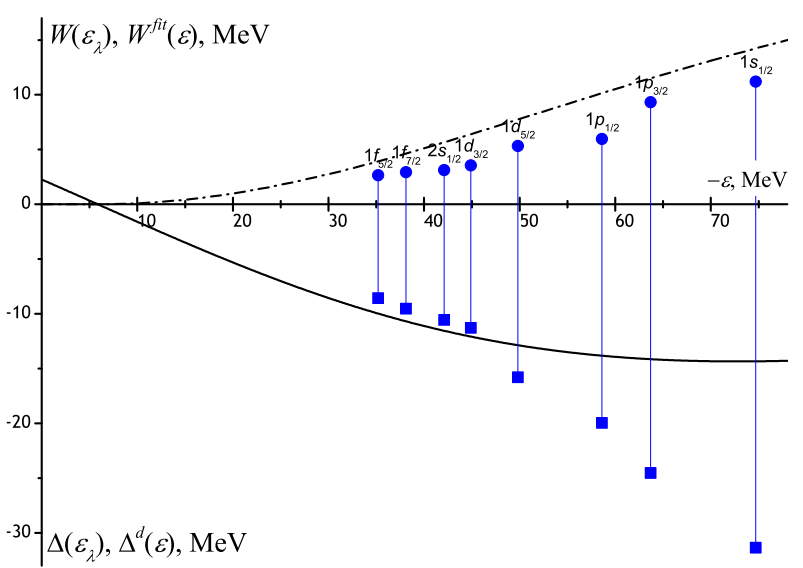

a)

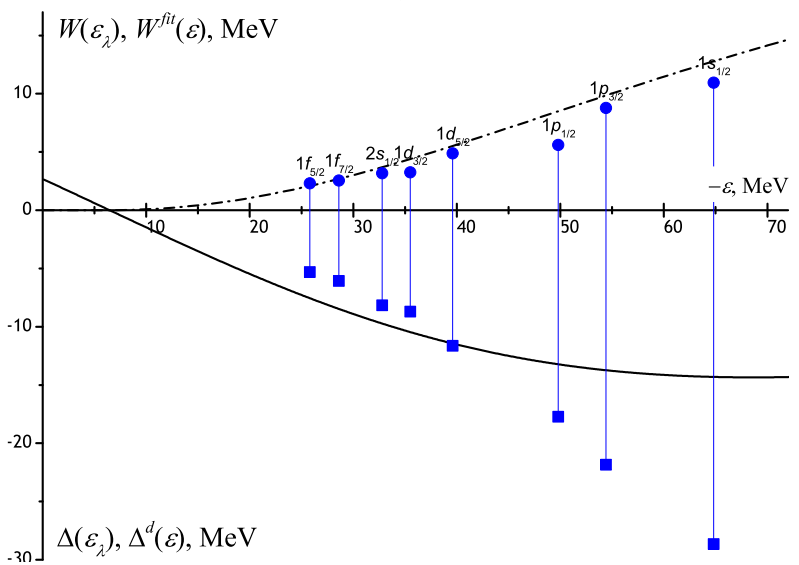

b)

Figure 3. (Color online) The same as in Fig. 2, but for the neutron (a) and proton (b) one-hole states in the ${ }^{208} \mathrm{~Pb}$ parent nucleus.

the second ("potential") term simulates the mean-field energy dependence. Using the above supposition for $W(|E|)$, one gets the dispersive relationship which determines the dispersive part of $\Delta$ via $W[3,4]$ :

$$
\Delta^{d}(E)=\frac{2 E}{\pi} P . V . \int_{0}^{\infty} \frac{W\left(E^{\prime}\right)}{E^{2}-E^{\prime 2}} d E^{\prime}
$$

To find the empirical function $W(|E|)$, we use, firstly, the experimental energies s-h states, $\varepsilon_{\lambda}$. Within the model, these energies can be calculated together with the s-h wave functions according to the equation

$$
\left\{H_{0}(x)+\Delta\left(\varepsilon_{\lambda}\right) f(x)-\varepsilon_{\lambda}\right\} \phi_{\lambda}(x)=0 .
$$

The wave functions, $\phi_{\lambda}(x)$, are supposed to be normalized to unity. Thus, we can find a set of the empirical quantities $\Delta\left(\varepsilon_{\lambda}\right)$. As shown in Ref. [3], the function $W(\varepsilon)$ determines the spreading (in fact, total) width of s-h states according to the relationship

$$
\Gamma_{\lambda}=2 W\left(\varepsilon_{\lambda}\right) \int f(x)\left|\phi_{\lambda}(x)\right|^{2} d x .
$$

Using Eq.(4) together with the experimental values of s-h total widths, we can find a set of quantities $W\left(\varepsilon_{\lambda}\right)$. 
Such a set can be adopted by an empirical function $W^{f i t}(|E|)$ which is proportional to $E^{2}$ for small $|E|$, as it takes place in infinite Fermi-systems. In accordance with Eq. (2), the adopted function $W^{f i t}(|E|)$ determines the dispersive part of the optical-model potential, $\Delta^{d}(\varepsilon)$. Being compared with the values $\Delta(\varepsilon)$, this quantity determines contribution of the spreading effect to the s-h excitation energies.

\section{Calculation results}

The sets of quantities $\Delta\left(\varepsilon_{\lambda}\right)$ and $W\left(\varepsilon_{\lambda}\right)$ found by the abovedescribed way are shown in Figs. 2, 3 for neutron and proton subsystems of ${ }^{90} \mathrm{Zr}$ and ${ }^{208} \mathrm{~Pb}$. The adopted functions $W^{f i t}(|E|)$ are parameterized as follows:

$$
W^{f i t}(|E|)=W_{0} \tanh ^{2}(E / B)
$$

with the adjusted parameters $W_{0}^{p}=25, W_{0}^{n}=20$ and $B^{p}=60, B^{n}=70$ (in $\mathrm{MeV}$ ). The functions $W^{\text {fit }}(|E|)$ are also shown in Figs. 2, 3. Finally, the dispersive part of the optical-model potential, $\Delta^{d}(\varepsilon)$, is evaluated by means of Eq. (2). For $\varepsilon<\mu$ these functions are shown in Figs. 2, 3 and compared with the sets of quantities $\Delta\left(\varepsilon_{\lambda}\right)$. From these comparison it follows that the spreading effect gives significant contribution to the excitation energy of deep-hole states.

In conclusion, we demonstrate abilities of the singlequasiparticle dispersive optical model to describe the spreading effect contribution to the excitation energy of deep-hole states in medium-heavy-mass spherical nuclei.

The authors are grateful to S. Shlomo for providing the data shown in Fig. 1.

\section{Acknowledgements}

This work is partially supported by Russian Foundation for Basic Research (grant No. 15-02-08007) and the Competitiveness Program of NRNU "MEPhI" (M. H. U.).

\section{References}

[1] A.A. Vorobyev et al., Phys. At. Nucl. 58, 1817 (1995)

[2] S. Shlomo, private communication

[3] G.V. Kolomiytsev, S.Yu. Igashov, and M.H. Urin, Phys. At. Nucl. 77, 1105 (2014)

[4] C. Mahaux and R. Sartor, Adv. Nucl. Phys. 20, 1 (1991) 